\title{
Experimental Highlights of the RHIC Program
}

\author{
Patricia Fachini
}

Brookhaven National Laboratory, P.O. BOX 5000, Upton, NY, USA.

\begin{abstract}
Experimental highlights of the RHIC program are reviewed.
\end{abstract}
\section{INTRODUCTION}

Relativistic heavy-ion collisions provide a unique environment to study matter under extreme conditions of high temperature and energy density. RHIC (Relativistic Heavy Ion Collider) at the Brookhaven National Laboratory in Upton, NY provides us with such collisions. Since the beginning of the RHIC operation in the year 2000, RHIC has provided us not only with $\mathrm{Au}+\mathrm{Au}$ collisions at the top energy of $\sqrt{s_{N N}}=200 \mathrm{GeV}$, but also at $62 \mathrm{GeV}$. RHIC has also provided collisions of lighter systems such as $\mathrm{Cu}+\mathrm{Cu}$ at $\sqrt{s_{N N}}=200,62$, and $22.5 \mathrm{GeV}$, and finally the $p+p$ and $d+\mathrm{Au}$ collisions at $\sqrt{s_{N N}}=200$ $\mathrm{GeV}$ that were used as references.

The amount of data obtained so far is as overwhelming as the results. I will try to summarize where we are, with RHIC RUNV just about to start.

\section{ELLIPTIC FLOW}

In non-central collisions the initial spatial anisotropy is transformed into an anisotropy in momentum-space if sufficient interactions occur among the constituents within the system. Once the system has expanded enough to quench the spatial anisotropy, further development of momentum anisotropy ceases. This self-quenching process happens quickly, so elliptic flow is primarily sensitive to the early stages of the collisions [1].

\section{Hydrodynamics}

The elliptic flow $v_{2}$ as a function of $p_{t}$ for $K_{S}^{0}, \Lambda, \phi, \Xi$, and $\Omega$ is depicted in Fig. 1 [2, 3]. The $\phi, \Xi$, and $\Omega$ have low hadronic cross-sections, therefore the large $v_{2}$ observed suggest that the elliptic flow is built up in the partonic stage. The expected range of $v_{2}$ from hydrodynamic calculations is also shown in Fig. 1 A more detailed comparison can be seen in Fig. 2 (left panel), where the mass dependence hydrodynamic results [1] are compared to the $v_{2}$ measurements of $\pi, K, p$, and $\Lambda$ [4]. Hydrodynamics describes well the mass dependence observed in the data that is characteristic of a common flow velocity. Since an ideal hydrodynamic fluid is a thermalized system with a zero mean 


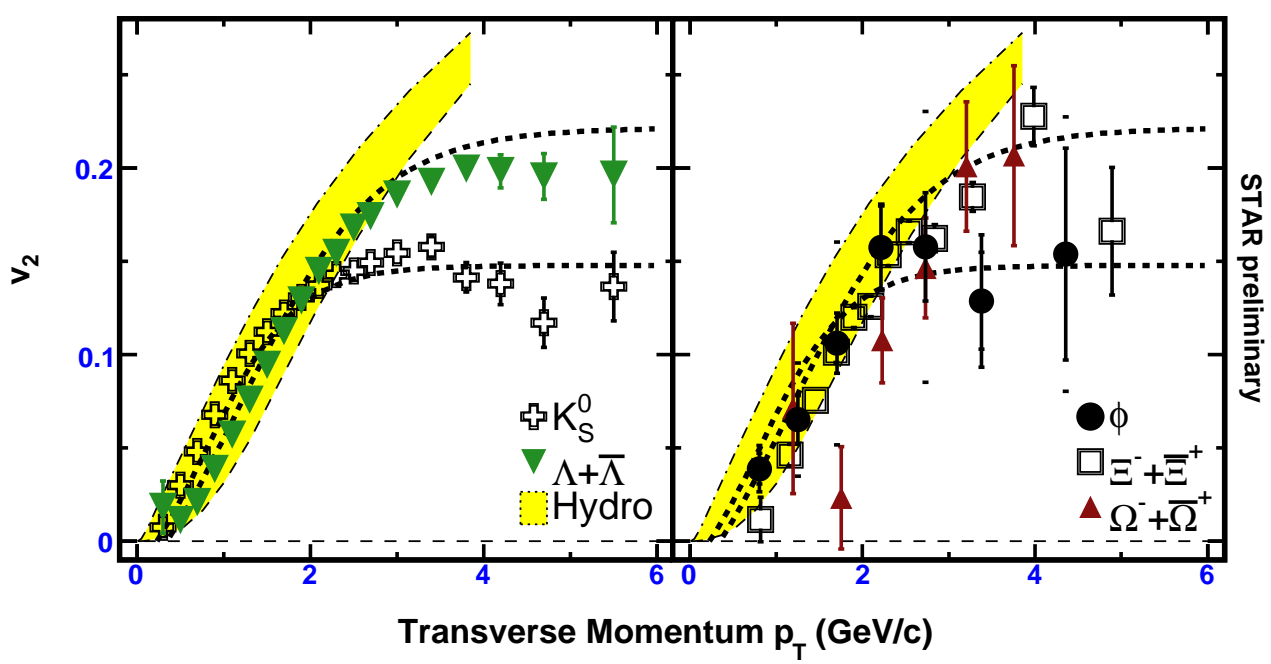

FIGURE 1. Azimuthal anisotropy $v_{2}$ for strange (left panel) and multi-strange (right panel) hadrons in minimum bias $\mathrm{Au}+\mathrm{Au}$ collisions [2]. Data measured by STAR. The dashed lines show a common fit to the $K_{S}^{0}$ and $\Lambda+\bar{\Lambda}$ data [5]. The shaded areas are hydrodynamic calculations [1]

free path that yields to the maximum possible $v_{2}$, the good agreement between the measured $v_{2}$ and the hydro results [1] suggests thermalization in heavy-ion collisions at RHIC.

\section{Constituent Quark Scaling}

While hydrodynamic calculations keep increasing as a function of $p_{T}$, the measured $v_{2}$ saturate at $p_{T}>2 \mathrm{GeV} / c$ [2]. The saturation value for mesons is about $2 / 3$ of that for baryons. This separation pattern holds for $\pi, K, \Lambda$, and $\Xi$, and seems to hold for $\phi$ and $\Omega[3,6]$. This result and the baryon-meson splitting of the high $p_{T}$ suppression pattern [7] suggest the relevance of the constituent quark degrees of freedom in the intermediate $p_{T}$ region [8]. $v_{2}$ scaled by the number of valence quarks $n$ as a function of $p_{T} / n$ is depicted in Fig. 2](right panel). The lower panel of Fig. 2](right panel) displays the ratio between the measurements and a polynomial fit to all the data. At low $p_{T} / n$ the observed deviations from the fit follow a mass ordering which is expected from hydrodynamics. At higher $p_{T}$, all $v_{2} / n$ measurements are reasonably close to unity showing the constituent quark scaling.

\section{FREEZE-OUT PROPERTIES}

The measured particle spectra and yields [9] and event-by-event $\left\langle p_{T}\right\rangle$ fluctuations [10] indicate a nearly chemically and kinetically equilibrated system at the final freeze-out stage. 

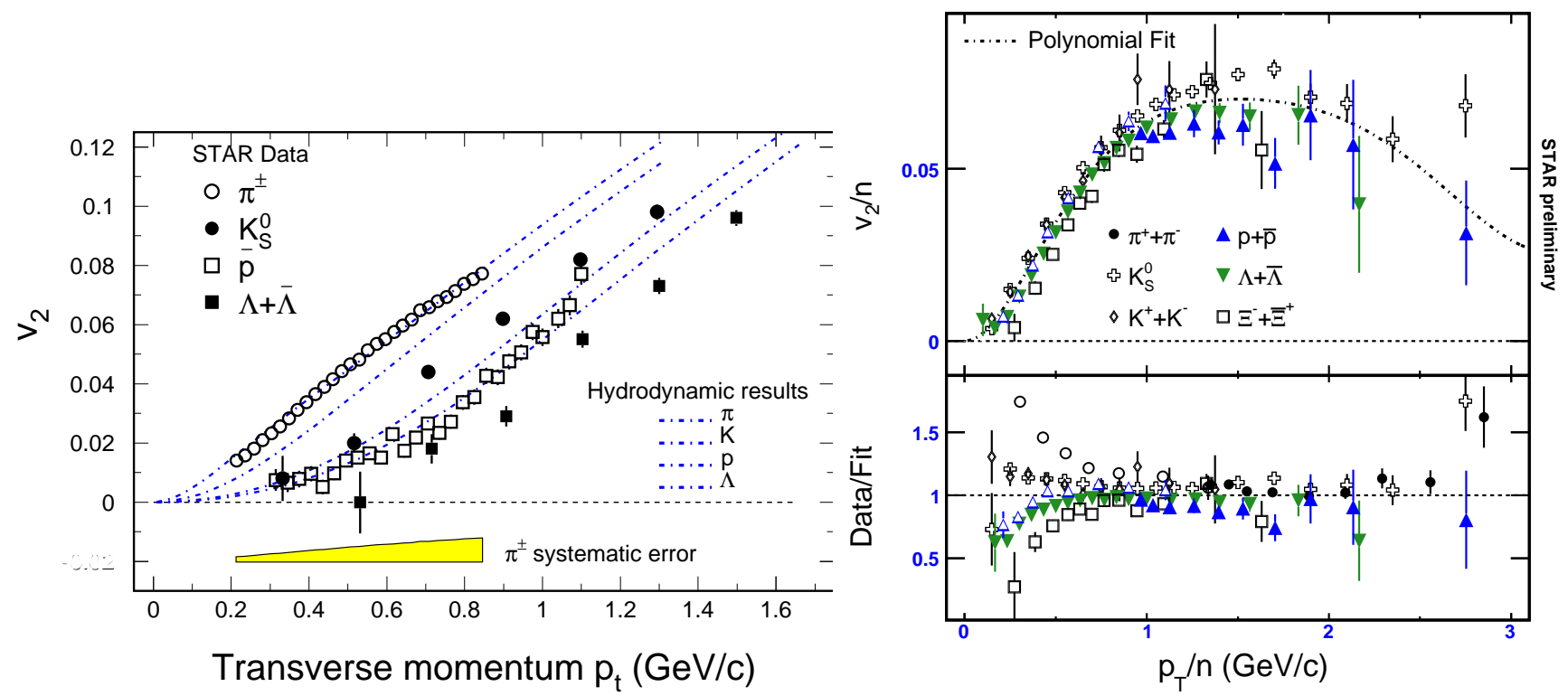

FIGURE 2. Left panel: $v_{2}$ as a function of $p_{t}$ from minimum bias Au+Au collisions [4] measured by STAR. The dotted-dashed lines are hydrodynamic calculations using an equation of estate (EOS) with a first-order hadron quark-gluon plasma phase transition [1]. The description of the data worsen if a hadronic EOS is used [1]. Right panel: Measurements by STAR of the scaled $v_{2}\left(p_{T} / n\right) / n$ for identified hadrons (upper panel) and the ratio between the measurements and a polynomial fit through all data points (lower panel) except the pions for $\sqrt{s_{N N}}=200 \mathrm{GeV}$ minimum bias $\mathrm{Au}+\mathrm{Au}$ collisions [2].

\section{Chemical and Kinetic Freeze-out Parameters}

STAR has now measured hadron distributions at $\sqrt{s_{N N}}=200$ and $62 \mathrm{GeV}$ 11, 12]. Chemical freeze-out properties were extracted from stable particle ratios within the thermal model [15]. The extracted chemical freeze-out temperature $T_{c h}$ and the strangeness suppression factor $\gamma_{s}$ are depicted in Fig. 3, Kinetic freeze-out properties from particle $p_{T}$ distributions were extracted within the blast wave model [16]. Figure [4 displays the extracted kinetic freeze-out temperature $T_{k i n}$ and the average radial flow velocity $\left\langle\beta_{T}\right\rangle$. The results at $\sqrt{s_{N N}}=62 \mathrm{GeV}$ are found to be qualitatively the same as those obtained at $\sqrt{s_{N N}}=200 \mathrm{GeV}$ and resonance decays are found to have no significant effect on the extract kinetic freeze-out parameters [12].

$T_{c h}$ is independent of centrality. $T_{\text {kin }}$ obtained from $\pi, K$, and $p$ decreases as a function of centrality, while the corresponding $\left\langle\beta_{T}\right\rangle$ increases. This is evidence that the system expands between chemical and kinetic freeze-outs, which brings the system to a lower temperature. The constant $T_{c h}$ suggests that hadronic scatterings from hadronization to chemical freeze-out may be negligible because they would result in a dropping $T_{c h}$.

$\gamma_{s}$ increases from $p+p$ to peripheral and central $\mathrm{Au}+\mathrm{Au}$ collisions. In central $\mathrm{Au}+\mathrm{Au}$ collisions, $\gamma_{s}$ is $\sim 1$ suggesting that strangeness is saturated. The $T_{c h}$ of $\phi, \Xi$, and $\Omega$ is higher than that of $\pi, K$, and $p$, while the $\left\langle\beta_{T}\right\rangle$ is lower. Noting that the $\phi$, $\Xi$, and $\Omega$ have small hadronic cross-sections, they may chemically and kinetically freeze-out at the same time. 

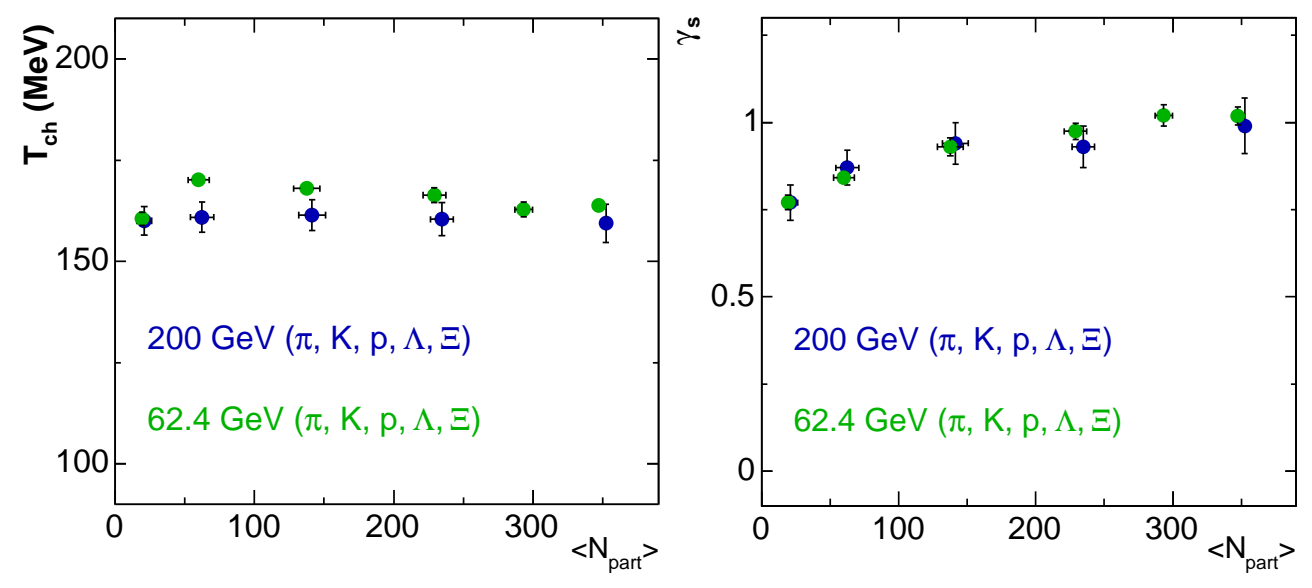

FIGURE 3. Left panel: Extracted chemical freeze-out temperature from stable particle ratios [11, 12].Right panel: Extracted strangeness suppression factor from stable particle ratios [11, 12]. STAR measurements.

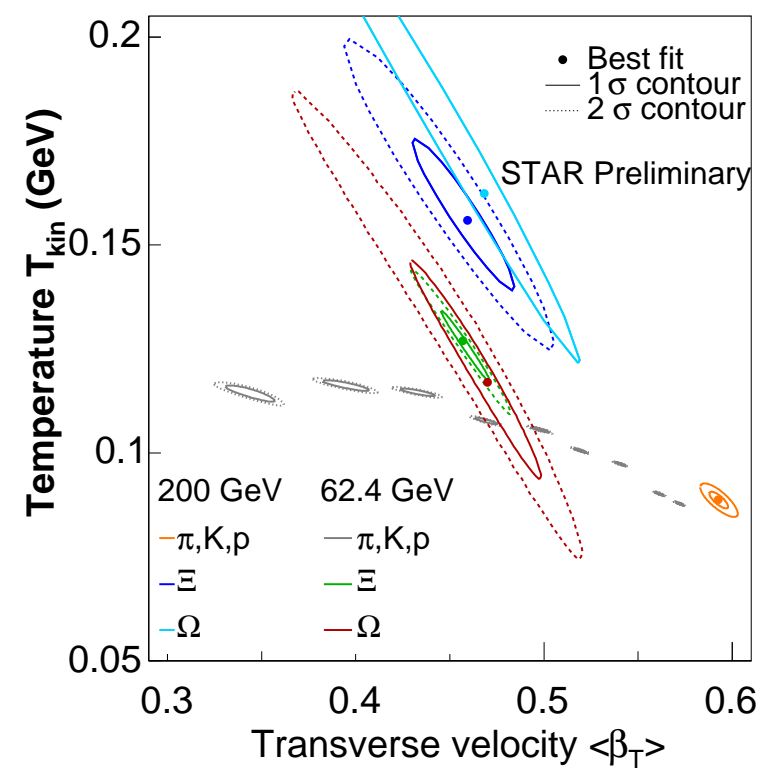

FIGURE 4. Extracted kinetic freeze-out temperature as a function of the average flow velocity within the blast-wave model [11, 12]. STAR measurements.

\section{SYSTEM SIZE DEPENDENCE}

The comparison between the charged hadron pseudorapidity distributions measured by PHOBOS [13] in $\mathrm{Cu}+\mathrm{Cu}$ and $\mathrm{Au}+\mathrm{Au}$ with similar number of participants $N_{\text {part }}$ is presented in Fig. 5] Both distributions are comparable within errors, showing that bulk particle production depends mainly on the number of participating nucleons. The same is true for different $N_{\text {part }}$ and also at $\sqrt{s_{N N}}=62 \mathrm{GeV}$ [13]. Many other measurements from BRAHMS, PHENIX, PHOBOS, and STAR support this argument [20, 30, 13, 7]. 


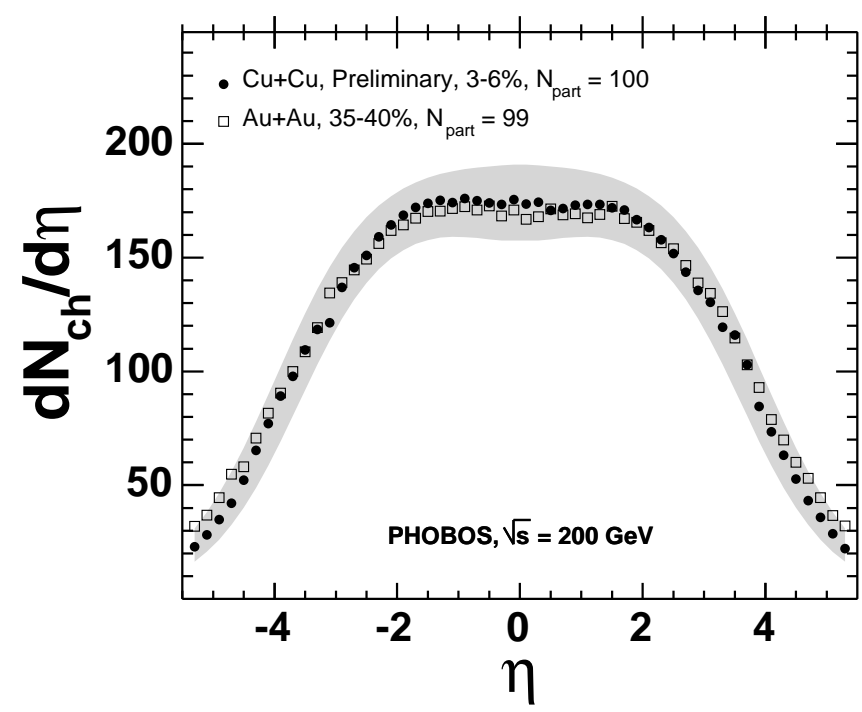

FIGURE 5. Charged hadron pseudorapidity measured by PHOBOS for $\mathrm{Cu}+\mathrm{Cu}$ and $\mathrm{Au}+\mathrm{Au}$ collisions at $\sqrt{s_{N N}}=200 \mathrm{GeV}$ with similar number of participants $N_{\text {part }}$

\section{LOW $P_{T}$ HADRONS}

The nuclear modification factor $R_{A A}$ of $\pi$ and $p$ measured at $y=3.2$ by BRAHMS and $y=0$ by PHENIX in central $\mathrm{Au}+\mathrm{Au}$ collisions does not depend on rapidity [20], suggesting that the same mechanisms is responsible for the nuclear modifications. It has been predicted [21] that the magnitude of jet quenching should depend on both the size and the density of the created absorbing medium. The averaged pion $R_{A A}$ as a function of $N_{\text {part }}$ for both forward rapidity and mid-rapidity are shown in Fig. 6. The average was performed in the interval $2<p_{T}<3 \mathrm{GeV} / c$. The mid- and forward rapidity pion suppression for the most central $\mathrm{Au}+\mathrm{Au}$ collisions are found to be the same magnitude. However, the $R_{A A}$ measured in forward rapidity shows significantly stronger rise towards peripheral collisions as compared to $R_{A A}$ at mi-rapidity, differing on the level of $35 \%$ for $\left\langle N_{\text {part }}\right\rangle \approx 100$. This is consistent with the model of parton energy loss in a strongly absorbing medium [22, 23]. In this picture, at mid-rapidity, the emission is dominated by the emission from the surface which quenches the dependence of $R_{A A}$ on the system. On the other hand, at forward rapidities, the transition from surface to volume emission can occur, which leads to a stronger dependence on the number of participants.

\section{INTERMEDIATE $P_{T}$ HADRONS}

Intermediate $p_{T}\left(p_{T}<6 \mathrm{GeV} / c\right)$ protons behave differently than mesons at heavy-ion collisions [6]. This behavior can be seen in Fig.7](left panel) that depicts the ratio of $p$ to $\pi$ spectra measured by STAR in both central Au+Au collisions and in $p+p$ collisions. The large enhancement of the $p / \pi$ ratio at intermediate $p_{T}$ in $\mathrm{Au}+\mathrm{Au}$ collisions indicates that jet fragmentation in vacuum is not the dominant source of particle production in 


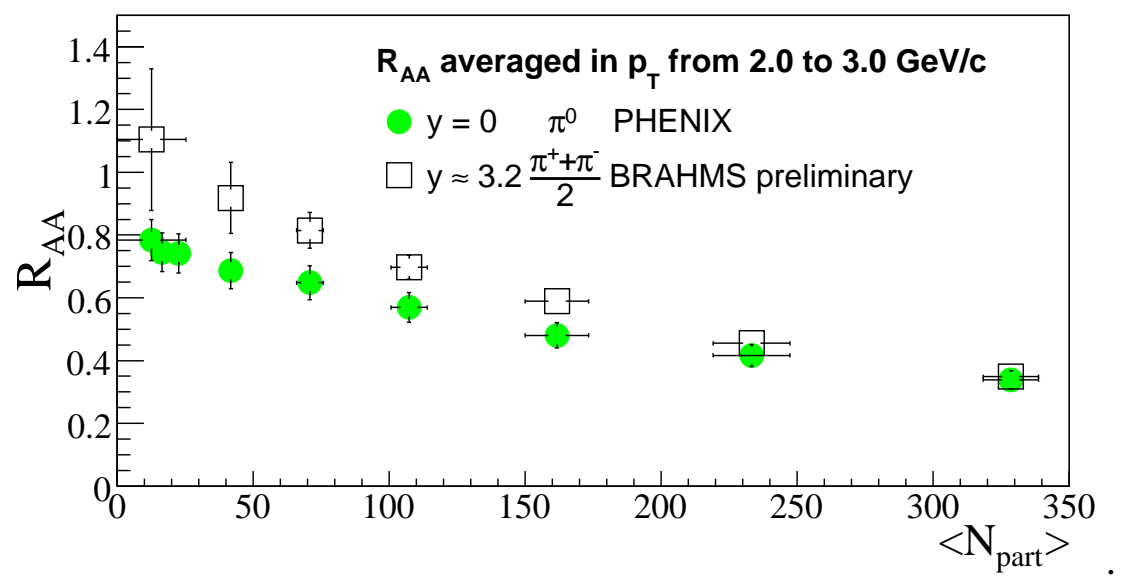

FIGURE 6. Averaged $R_{A A}$ in the interval $2.0<p_{T}<3.0 \mathrm{GeV} / c$ at mid-rapidity (PHENIX) and at forward rapidity (BRAHMS) as a function of number of participants at $\sqrt{s_{N N}}=200 \mathrm{GeV}$ [20]

this $p_{T}$ range. An enhancement, roughly peaked at at the same position, is also observed in $R_{C P}$, as shown in Fig. 7 (right panel). Here, $R_{C P}$ is the ratio between central and peripheral $\mathrm{Au}+\mathrm{Au}$ collisions normalized to the binary collision scaling expectation. Like $v_{2}$ [2], $R_{C P}$ also separates into baryons and mesons. The $K^{*}$ [17], which is a meson with the mass close to the proton mass, follow the behavior of mesons. This proves that this separation is not due to the hadron mass. This grouping is violated in $R_{A A}$, where the reference is from $p+p$ collisions rather than peripheral collisions. A strong enhancement in strange baryon $R_{A A}$ with increasing enhancement for increasing strangeness content is observed at intermediate $p_{T}$ [18]. This enhancement is depicted in Fig. 8 .

\section{HIGH $P_{T}$ HADRONS}

The suppression of high $p_{T}$ hadrons in central $A u+A u$ collisions was one of the unexpected and important phenomena observed at RHIC. The $R_{A A}$ of $\pi^{0}$ measured by PHENIX in central Au+Au collisions at $\sqrt{s_{N N}}=200 \mathrm{GeV}$ [24] is presented in Fig. 9 The suppression is quite strong $\left(R_{A A} \sim 0.2\right)$ and remains approximately flat up to 20 $\mathrm{GeV} / c$. Partonic radiative energy loss models [25, 26, 27] reproduce this behavior well. In particular, one calculation depicted in Fig. 9 uses an average gluon average density $d N_{g} / d y \sim 1200$ [25]. The $R_{A A}$ measured by PHENIX and STAR are shown in Fig. 9 [24] and Fig. 10 [19], respectively. The difference between charged hadrons $R_{A A}$ and the $\pi^{0} R_{A A}$ in the intermediate $p_{T}$ region $\left(p_{T}<6 \mathrm{GeV} / c\right)$ is due to the proton contribution as observed in Fig. 10 and nicely described by recombination models. This behavior disappears for $p_{T}>6 \mathrm{GeV} / c$ and are also explained by the same recombination models [28, 29, 30]. 

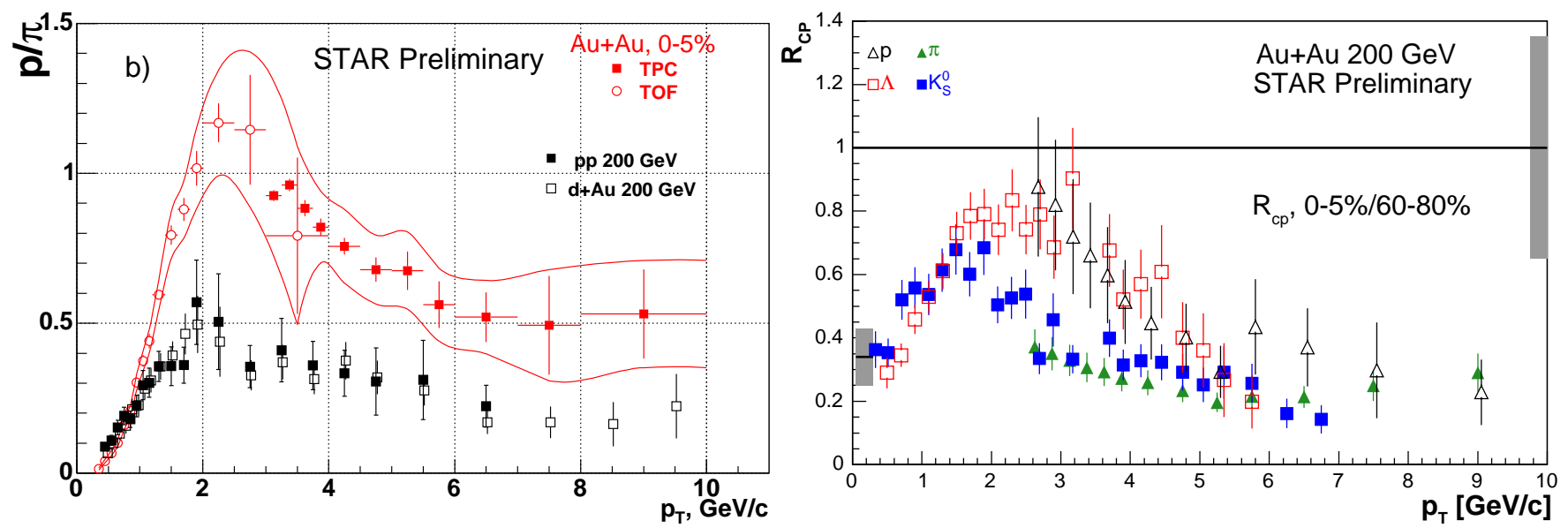

FIGURE 7. Left panel: $p / \pi$ ratio measured by STAR as a function of $p_{T}$ for central Au+Au $(0-5 \%)$, $d+\mathrm{Au}$ and $p+p$ collisions [19]. For the Au+Au measurements, the error bars are statistical only and the solid lines are the systematic uncertainties. For the $d+\mathrm{Au}$ and $p+p$ measurements, the errors shown are combined statistical and systematic. Right panel: $R_{C P}$ as a function of $p_{T}$ for identified particles measured by STAR [18, 19]. Errors are statistical and systematic. Grey bands are common scale uncertainties from $N_{\text {binary. }}$

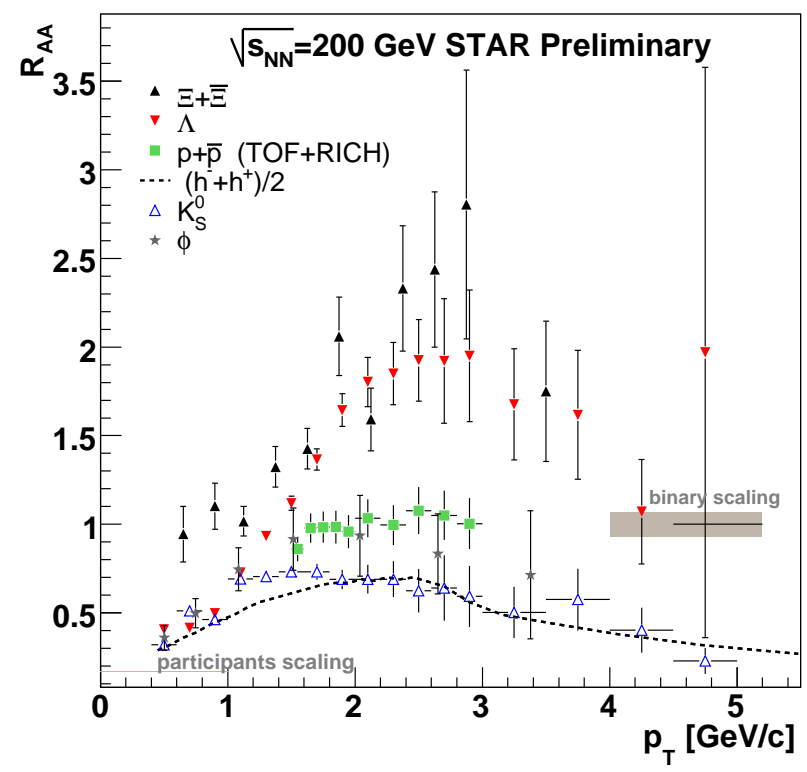

FIGURE 8. $\quad R_{A A}$ as a function of $p_{T}$ for identified particles measured by STAR [18, 19]. Errors are statistical and systematic. Grey bands are common scale uncertainties from $N_{\text {binary }}$.

\section{JET CORRELATIONS}

A well defined back-to-back peak that is characteristic of di-jets is observed with negligible background in Fig. 11 in both peripheral and central Au+Au collisions measured by STAR [31]. While the yield is substantially less in central Au+Au collisions, the widths 


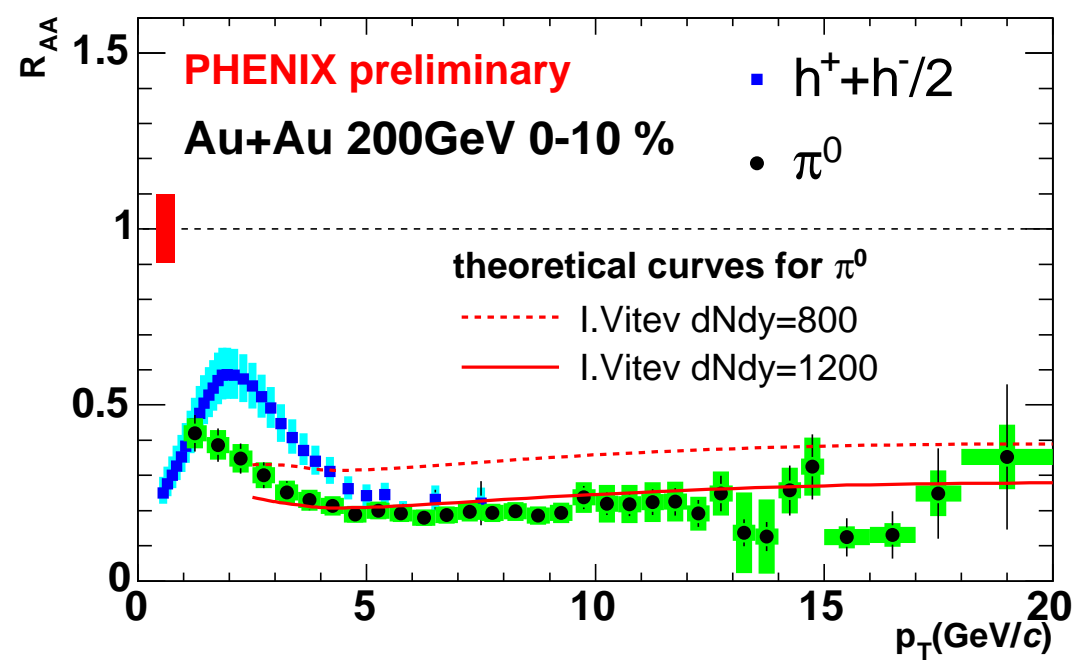

FIGURE 9. $R_{A A}$ as a function of $p_{T}$ of $\pi^{0}$ and charged hadrons measured by PHENIX in central Au+Au collisions $(0-10 \%)$ with theoretical predictions [25, 26]. The shaded areas are the systematic uncertainties.

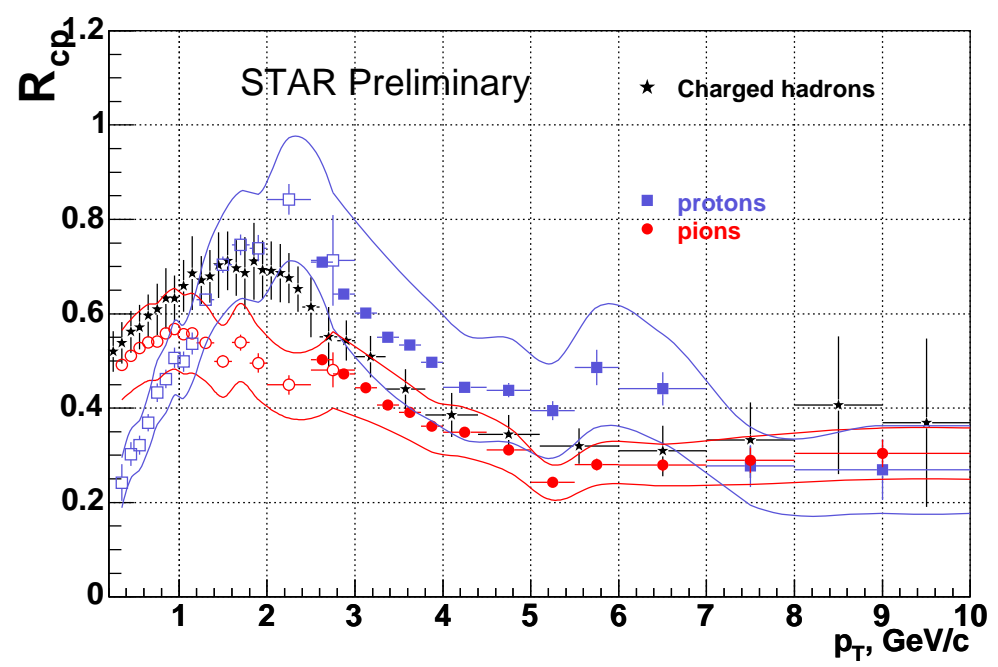

FIGURE 10. $R_{C P}$ for the $5 \%$ most central collisions measured by STAR, normalized by the peripheral $60-80 \%$ collisions, as a function of $p_{T}[19]$. The bands show combined statistical and systematic errors.

of the back-to-back peaks appear to be independent of centrality. The away-side hadron triggered fragmentation functions [26] for $d+\mathrm{Au}$ and $\mathrm{Au}+\mathrm{Au}$ collisions [31] measured by STAR as a function of $z_{T}=p_{T}^{\text {assoc }} / p_{T}^{\text {trig }}$ is depicted in Fig.12 (left panel). Fig. 12 (left panel) shows that the shape of the away-side fragmentation functions is unchanged from $d+\mathrm{Au}$ to central $\mathrm{Au}+\mathrm{Au}$ collisions. However, the yields are reduced by a factor of $\sim 4$ in central $\mathrm{Au}+\mathrm{Au}$ collisions. Even though the shape is consistent with previous predictions in the $z_{T}$ range measured, the magnitude is smaller than expected [26]. A different calculation predicted that significant energy loss should be associated with significant broadening of the away-side hadron azimuthal distribution [32], in contradiction to the 


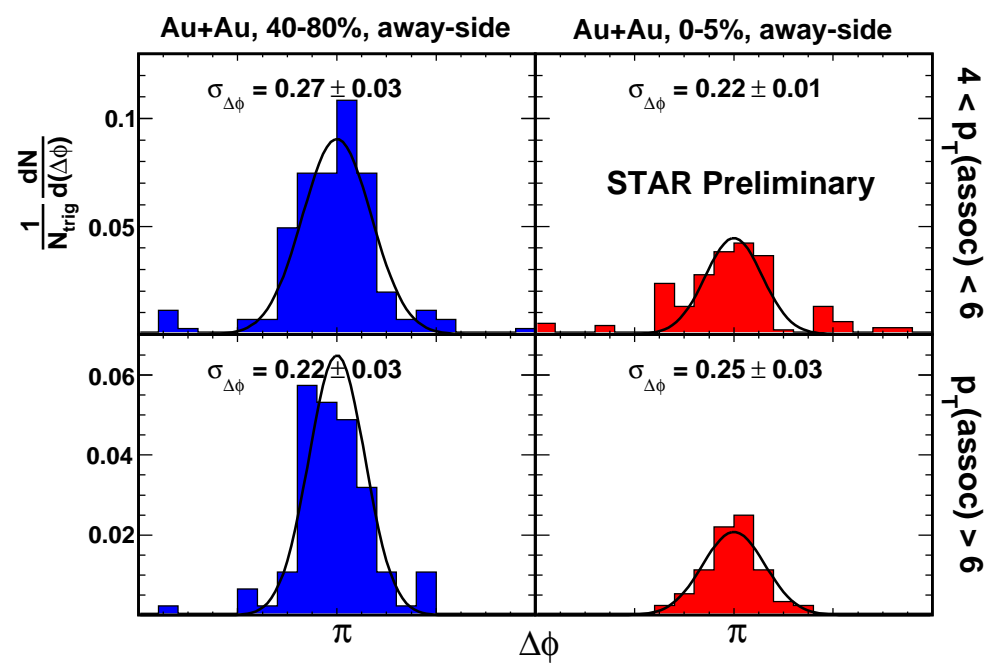

FIGURE 11. Azimuthal distributions of away-side charged hadrons for $8<p_{T}^{\text {trig }}<15 \mathrm{GeV} / c$ in two different centralities at $\sqrt{s_{N N}}=200 \mathrm{GeV} \mathrm{Au}+\mathrm{Au}$ collisions. These are the total distributions, no backgrounds have been subtracted.

STAR measurements.

When low- $p_{T}$ associated particles are observed in central Au+Au collisions opposite a high- $p_{T}$ trigger particle, they are found to be significantly broadened in $\Delta \phi$ and enhanced in number compared to $p+p$ collisions [33]. Contrary to the maximum found in $p+p$ and $d+\mathrm{Au}$ collisions, STAR measured the $\left\langle p_{T}\right\rangle$ of the associated particles appears to be a minimum at $\Delta \phi=\pi$ in central $\mathrm{Au}+\mathrm{Au}$ collisions [34]. In addition, the awayside associated particle yield is flat or may have a small dip at $\Delta \phi=\pi$ [34]. These phenomena are displayed in Fig. 12 (right panel) and have led to predictions that we may be observing jets that have been deflected by radial flow or a Mach cone effect associated with conical shock waves [35].

\section{HEAVY FLAVOR}

The nuclear modification factor $R_{A A}$ of non-photonic electrons measured by PHENIX [36] and STAR [7] in central Au+Au collisions is shown in Fig. 13. Both experiments observe a very strong suppression of the non-photonic electrons. The suppression has approximately the same shape and magnitude as the suppression for hadrons, which is a quite surprising result, since the massive quarks are expected to radiate much less energy than the lighter $u$ and $d$ quarks. It is important to confirm and complement these results with direct measurements of open charm and open bottom contributions. The first direct reconstruction of $D$ mesons by STAR [37] is the first step in this direction, and more improvement will come with the implementation of new detectors from both PHENIX and STAR.

The $v_{2}$ of non-photonic electrons as a function of $p_{T}$ [36] measured by PHENIX is shown in Fig. 14 (left panel). The non-zero flow measured at $p_{T}<2 \mathrm{GeV} / c$, where the 

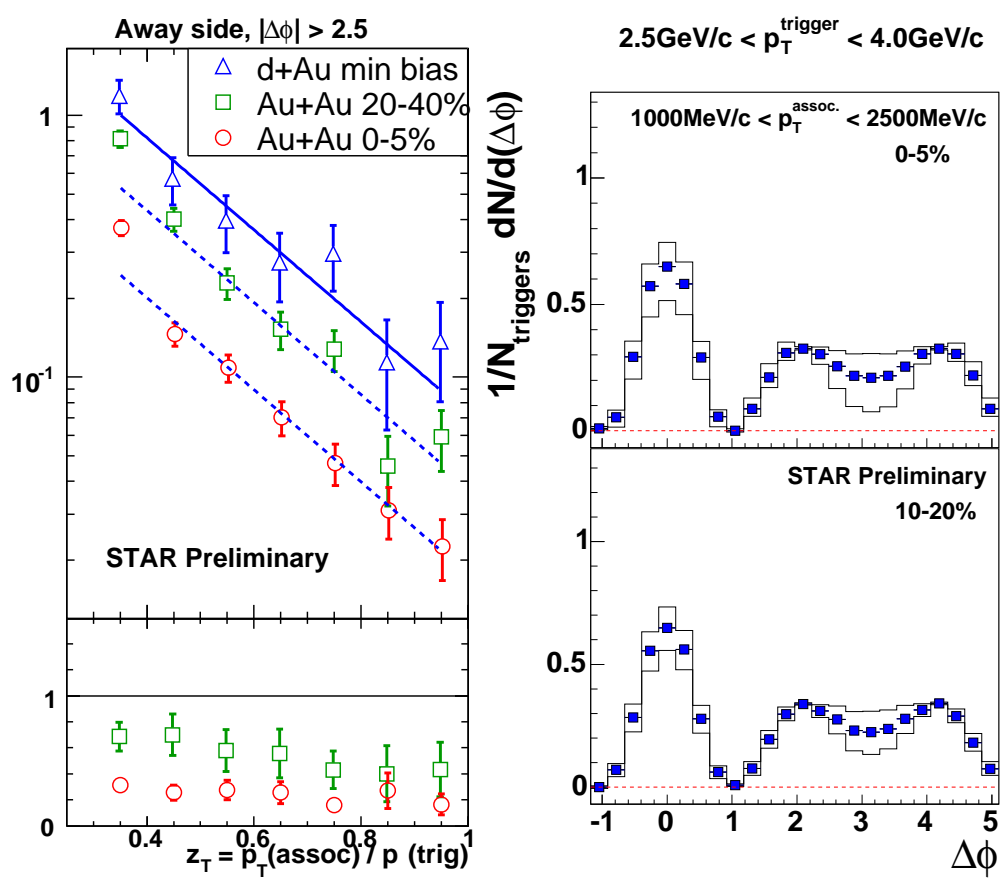

FIGURE 12. Left panel: Top: Hadron triggered fragmentation functions $d N / d z_{T}$ for away-side charged hadrons as a function of $z_{T}$ for $8<p_{T}^{\text {trig }}<15 \mathrm{GeV} / c$ at $\sqrt{s_{N N}}=200 \mathrm{GeV} d+\mathrm{Au}$ and Au+Au collisions. The solid line is an exponential fit to the $z_{T}$ distribution for $d+\mathrm{Au}$. The dashed lines represent the same exponential fit scaled down by factors of 0.54 and 0.25 to approximate the yields in $20-40 \%$ and $0-5 \%$ $\mathrm{Au}+\mathrm{Au}$ collisions. Bottom: Ratio of the hadron triggered fragmentation functions for $\mathrm{Au}+\mathrm{Au} / d+\mathrm{Au}$. Right panel: Azimuthal distributions of associated charged hadrons for two different centralities at $\sqrt{s_{N N}}=200$ $\mathrm{GeV} \mathrm{Au+Au} \mathrm{collisions.} \mathrm{The} \mathrm{histograms} \mathrm{indicate} \mathrm{the} \mathrm{systematic} \mathrm{uncertainty} \mathrm{bands.}$
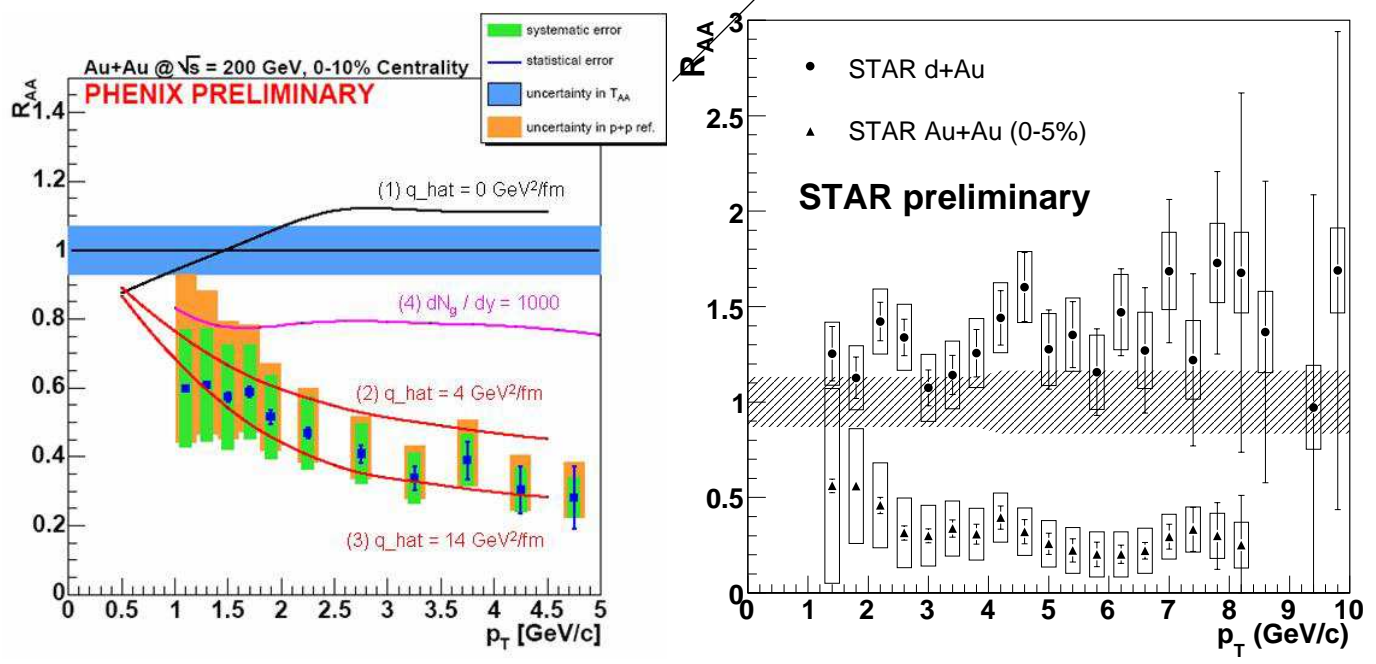

FIGURE 13. Left panel: $R_{A A}$ of non-photonic electrons measured by PHENIX [36] for central $\mathrm{Au}+\mathrm{Au}$ collisions at $\sqrt{s_{N N}}=200 \mathrm{GeV}$. Right panel: $R_{A A}$ of non-photonic electrons measured by STAR [7] for central $\mathrm{Au}+\mathrm{Au}$ collisions at $\sqrt{s_{N N}}=200 \mathrm{GeV}$. 

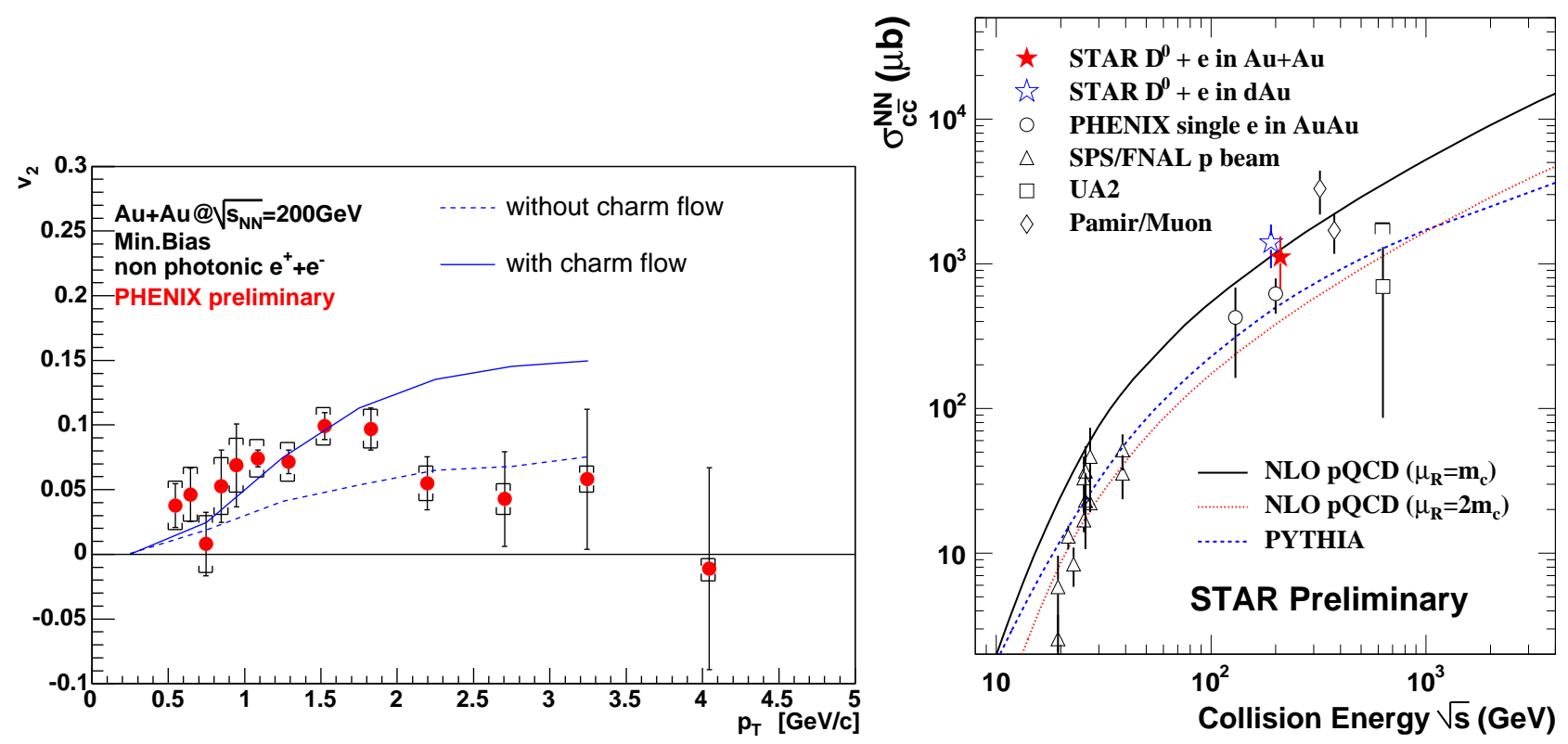

FIGURE 14. Left panel: $v_{2}$ of non-photonic electrons attributted to semi-leptonic open charm decays measured by PHENIX [36] and compared to theoretical predictions [38]. Right panel: Compilation of the total charm cross-section production per nucleon collision $\sigma_{c \bar{c}}^{N N}$ as a function of energy compared to the NLO pQCD calculations [37].

yield is dominated by semi-leptonic decays of open charm, suggests a sizeable flow of $D$ mesons. The comparison to calculations with and without charm flow [38] shown in Fig. 14 (left panel) favors the interpretation of charm flow. The consequence is a strong interaction with the medium and a high degree of thermalization of $c$ quarks, favoring the strongly coupled QGP.

PHENIX has calculated the non-photonic electron measurements in $p+p$ and minimum $\mathrm{Au}+\mathrm{Au}$ collisions at $\sqrt{s_{N N}}=200 \mathrm{GeV}$ into a total cross-section of charm production per nucleon collision $\sigma_{c \bar{c}}^{N N}$ [39]. STAR has calculated the combined electron and direct $D$ meson measurements in minimum bias $d+\mathrm{Au}$ and minimum bias $\mathrm{Au}+\mathrm{Au}$ collisions [37]. There is a factor of $\sim 2$ difference in their minimum bias $\mathrm{Au}+\mathrm{Au}$ crosssections. It is important to notice that STAR is sensitive to $80 \%$ of the total charm crosssection, while PHENIX is only sensitive to $15 \%$. A compilation of charm cross-sections $\sigma_{c \bar{c}}^{N N}$ as a function of collision energy is displayed in Fig. 14 (right panel). Results from PHENIX and STAR are also shown.

\section{$\mathbf{J} / \psi$}

PHENIX measures $J / \psi$ in $p+p, d+\mathrm{Au}, \mathrm{Cu}+\mathrm{Cu}$, and $\mathrm{Au}+\mathrm{Au}$ collisions at $\sqrt{s_{N N}}=200$ $\mathrm{GeV}$ and $\mathrm{Cu}+\mathrm{Cu}$ at $\sqrt{s_{N N}}=62 \mathrm{GeV}$ at mid-pseudorapidity $|\eta|<0.35$ through the $e^{+} e^{-}$ decay channel and forward pseudorapidity $|\eta| \in[1.2,2.2]$ through the $\mu^{+} \mu^{-}$decay channel [40]. The $R_{A A}$ as a function of centrality $N_{\text {part }}$ for the measurements at $\sqrt{s_{N N}}$ 

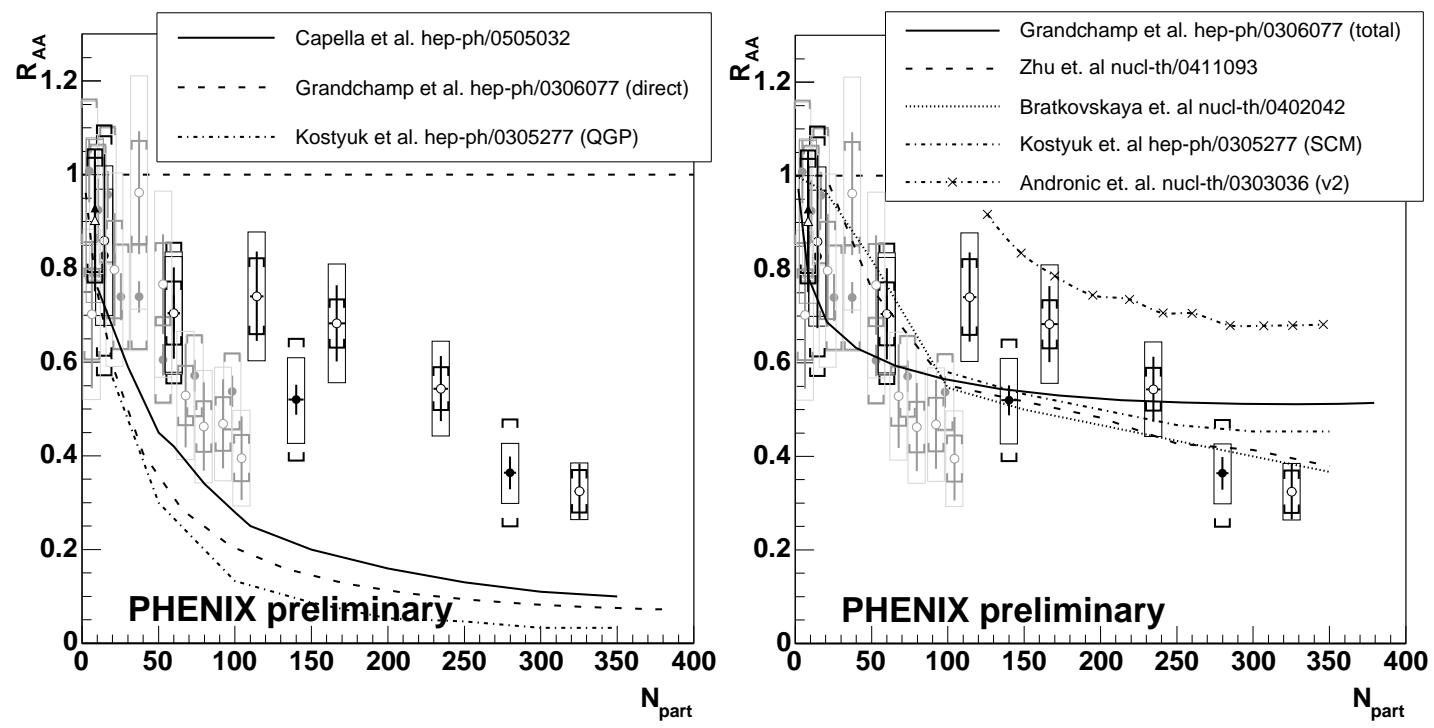

FIGURE 15. $\quad R_{A A}$ of $J / \psi$ as a function of $N_{\text {part }}$ in $d+\mathrm{Au}, \mathrm{Cu}+\mathrm{Cu}$, and $\mathrm{Au}+\mathrm{Au}$ collisions at $\sqrt{s_{N N}}=$ $200 \mathrm{GeV}$ measured by PHENIX. Left panel: The measurements are compared to models that explain the $J / \psi$ NA50 anomalous suppression [42, 43, 44]. Right panel: The measurements are compared to models involving either $J / \psi$ regeneration by quark recombination [43, 44, 45, 46] or $J / \psi$ transport in medium [47].

$=200 \mathrm{GeV}$ are depicted in Fig. 15. The suppression is clear and within errors it is seems to be independent of the collision system. Furthermore, the magnitude of the suppression of $\sim 3$ for the most central collisions is similar to the suppression observed at SPS [41].

The left panel of Fig. 15 shows that models that were able to explain the anomalous $J / \psi$ suppression at the SPS and that were based on interactions with comovers [42], color screening [43], or QCD-inspired in-medium effects [44] predict a stronger suppression at RHIC.

The discrepancy seems to be resolved by invoking the regeneration of $J / \psi$ at the later stage of the collision via recombination of $c$ and $\bar{c}$ quarks, which are produced more abundantly at RHIC. Several attempts that combine suppression and recombination [43, 44, 45, 46] reproduce the data reasonably well, as shown in the right panel of Fig. 15.

\section{LOW-MASS DI-ELECTRON}

The left panel from Fig. 16 depicts the measured di-electrons pairs, the background, and the subtracted spectra with uncertainty from PHENIX [48]. The right panel of Fig. 16 shows the data compared to the hadronic cocktail [48], and to theoretical predictions [49], where the $e^{+} e^{-}$invariant mass spectra have been calculated using different in-medium $\rho$ spectral functions and an expanding thermal fireball model. It is still premature for any statement, but it is definitely promising. 

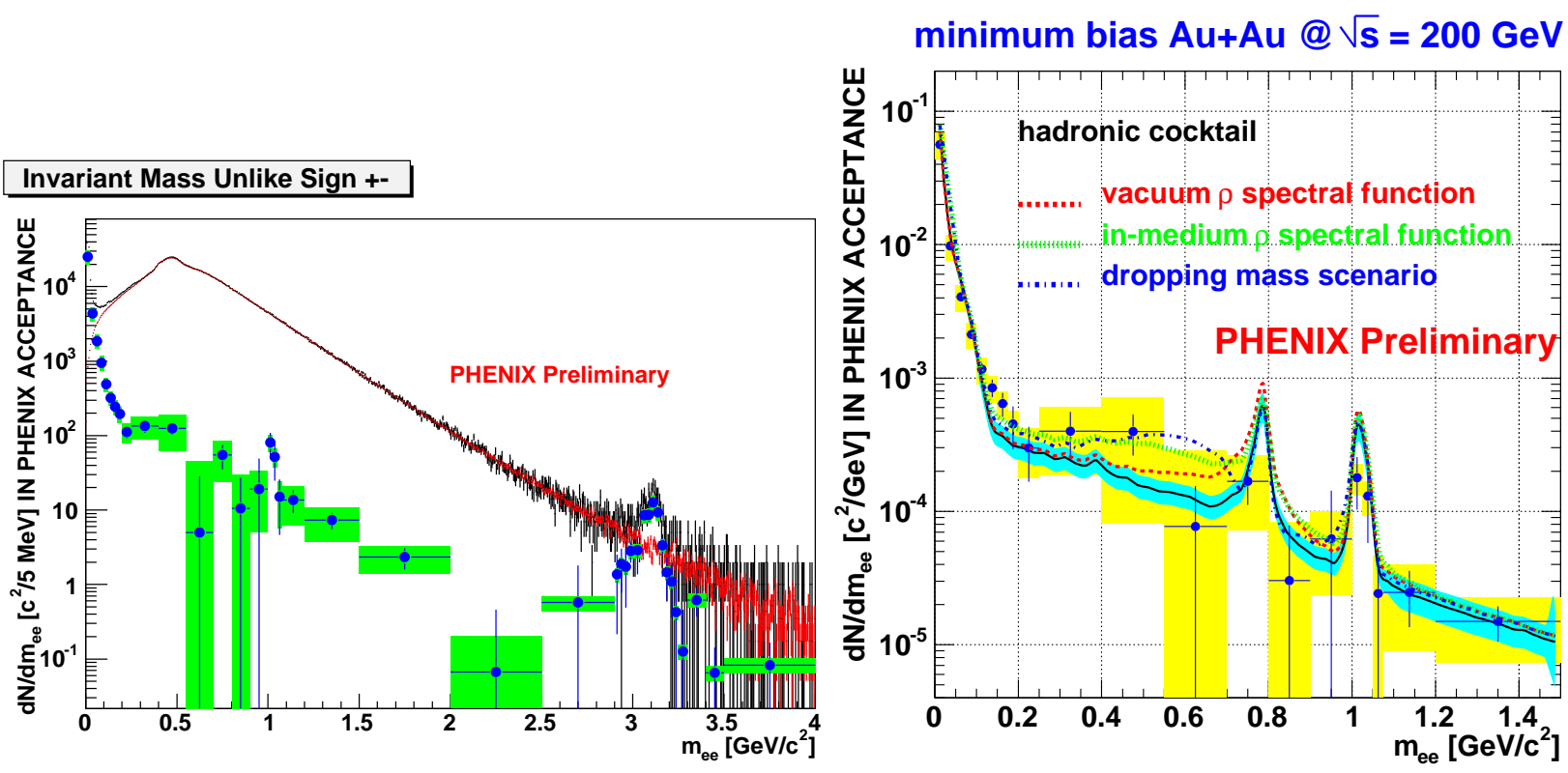

FIGURE 16. Left panel: The unlike sign mass spectrum together with the subtracted spectrum. Right panel: The data compared to the hadronic cocktail [48], and to theoretical predictions [49], where a $\rho$ spectral function is introduced with and without in-medium modifications.

\section{SUMMARY}

The baryon-meson effect observed at RHIC favors the constituent quark coalescence as the production mechanism at intermediate $p_{T}$, suggesting a partonic state prior to hadronization. $\phi, \Xi$, and $\Omega$ have small hadronic cross-sections, therefore the large $v_{2}$ observed suggest that it is built up in the partonic stage. Furthermore, the thermal parameters extracted from these particles suggest that they chemically and kinetically freeze-out at the same time.

Intermediate $p_{T}\left(p_{T}<6 \mathrm{GeV} / c\right)$ protons behave differently than mesons at heavyion collisions. The large enhancement of the $p / \pi$ ratio at intermediate $p_{T}$ in Au+Au collisions indicates that jet fragmentation in vacuum is not the dominant source of particle production in this $p_{T}$ range.

The suppression of high $p_{T}$ hadrons in central $A u+A u$ collisions was one of the unexpected and important phenomena observed at RHIC. The suppression is quite strong and remains approximately flat up to $20 \mathrm{GeV} / c$. Partonic radiative energy loss models [25, 26, 27] reproduce this behavior well.

The quality of the RHIC data is just marvellous, and the knowledge that this data has provided on the dense matter formed in relativistic heavy-ion collisions is without any doubt unexpected. We can only wonder how new data with new detector upgrades will surprise us. 


\section{ACKNOWLEDGMENTS}

I would like to thank F. Laue, R. Longacre, T. Ullrich, Z. Xu, and H. Zhang for the exciting discussions. This work was supported in part by the HENP Divisions of the Office of Science of the U.S. DOE.

\section{REFERENCES}

1. P. Huovinen, nucl-th/0305064 P. Kolb, and U. Heinz, nucl-th/0305084 E. V. Shuryak, Prog. Part. Nucl. Phys., 53, 273 (2004).

2. M. Oldenburg et. al., nucl-ex/0510026

3. X. Cai et. al., nucl-ex/0511004

4. J. Adams et. al., Phys. Rev. C 72, 014904 (2005).

5. X. Dong et. al., Phys. Lett. B 597, 328 (2004).

6. J. Adams et. al., Phys. Rev. Lett. 92, 052302 (2004); J. Adams et. al.,nucl-ex/0504022

7. J. Dunlop et. al., nucl-ex/0510073

8. D. Molnar and S. A. Voloshin, et. al., Phys. Rev. Lett. 91, 92301 (2003).

9. J. Adams et. al., Phys. Rev. Lett. 92, 112301 (2004).

10. J. Adams et. al., nucl-ex/0308033

11. L. Molnar et. al., nucl-ex/0507027

12. J. Speltz et. al., nucl-ex/0512037

13. G. Roland et. al., nucl-ex/0510042

30. V. Greene et. al., QM2006 Proceedings.

15. P. Braun-Munzinger, I. Heppe, and J. Stachel Phys. Lett. B, 465, 15 (1999); N. Xu and M. Kaneta, Nucl. Phys. A 698, 306 (2002).

16. E. Schnedermann, J.Sollfrank, and U. W. Heinz Phys. Rev. C, 48, 2462 (1993); U. A. Wiedemann and U. W. Heinz, Phys. Rev. C 56, 3265 (1997).

17. J. Adams et. al., Phys. Rev. C, 71, 064902 (2005);

18. S. Salur et. al., nucl-ex/0509036

19. O. Barannikova et. al., QM2005 Proceedings.

20. P. Staszel et. al., nucl-ex/0510061

21. M. Gyulassy, P. Levai, and I. Vitev, Nucl. Phys. B, 594, 594 (2001); Phys. Rev. D, 66, 014005 (2002).

22. A. Dainese, C. Loizides, and G. Paić, Eur. Phys. J. C, 38, 461 (2005).

23. A. Drees, H. Feng, and J. Jia, Phys. Rev. C, 71, 034909 (2005).

24. M. Shimomura et. al., nucl-ex/0510023

25. I. Vitev, Phys. Rev. Lett. 89, 252301 (2002).

26. X. N. Wang, Phys. Lett. B 595, 165 (2004).

27. K. Eskola et. al., Nucl. Phys. A 747, 511 (2005).

28. R. J. Fries et. al., Phys. Rev. Lett. 90, 202303 (2003); Phys. Rev. C, 68, 044902 (2003).

29. R. C. Hwa and C. B. Yang, Phys. Rev. C, 70, 024904 (2003).

30. V. GreKo, C. M. Ko, and P. Levai, Phys. Rev. C, 68, 034904 (2003).

31. D. Magestro et. al., nucl-ex/0510002

32. I. Vitev, Phys. Lett. B 630, 78 (2005).

33. J. Adams et. al., Phys. Rev. Lett. 95, 152301 (2005)

34. J. G. Ulery et. al., nucl-ex/0510055

35. H. Stoecker, Nucl. Phys. A 750, 121 (2005); J. Casalderrey-Solana, E. V. Shuryak, and D. Teaney, hep-ph/0411314, J. Ruppert and B. Muller, Phys. Lett. B 618, 123 (2005).

36. S. Butsyk et. al., nucl-ex/0510010

37. H. Zhang et. al., nucl-ex/0510063

38. V. GreKo, C. M. Ko, and R. Rapp, Phys. Rev. Lett., 68, 202 (2004).

39. S. S. Adler et. al., Phys. Rev. Lett., 94, 082301 (2005).

40. H. Buesching et. al., nucl-ex/0511044.

41. I. Tserruya et. al., nucl-ex/0601036 
42. A. Capella and E. G. Ferreiro, Eur. Phys. J. C, 42, 419 (2005).

43. A. P. Kostyuk et. al., Phys. Rev. C, 68, 041902 (2003).

44. L. GrandChamp, R. Rapp, and G. E. Brown, Phys. Rev. Lett., 92, 212301 (2004).

45. E. L. Bratkovskaya et. al., Phys. Rev. C, 69, 054903 (2004).

46. A. Andronic et. al., Phys. Lett. B 571, 36 (2003).

47. X. L. Zhu, P.F. Zhuang, and N. Xu, Phys. Lett. B 607, 107 (2005).

48. A. Toia, et. al., nucl-ex/0510006

49. R. Rapp, Phys. Rev. C, 63, 054907 (2001); nucl-th/0204003 